\title{
Pure Parsimony Xor Haplotyping
}

\author{
Paola Bonizzoni, Gianluca Della Vedovał Riccardo Dondił Yuri Pirola $§$ and Romeo Rizzi $\uparrow^{\Uparrow}$ \\ Preliminary version
}

\begin{abstract}
The haplotype resolution from xor-genotype data has been recently formulated as a new model for genetic studies 2 . The xor-genotype data is a cheaply obtainable type of data distinguishing heterozygous from homozygous sites without identifying the homozygous alleles. In this paper we propose a formulation based on a well-known model used in haplotype inference: pure parsimony. We exhibit exact solutions of the problem by providing polynomial time algorithms for some restricted cases and a fixed-parameter algorithm for the general case. These results are based on some interesting combinatorial properties of a graph representation of the solutions. Furthermore, we show that the problem has a polynomial time $k$-approximation, where $k$ is the maximum number of xor-genotypes containing a given SNP. Finally, we propose a heuristic and produce an experimental analysis showing that it scales to real-world large instances taken from the HapMap project.
\end{abstract}

\section{Introduction}

In this paper we investigate a computational problem arising in genetic studies of diploid organisms. In such organisms (which include all vertebrates), all chromosomes are in two copies, one inherited from the mother and one from the father. Since chromosomes are almost identical except for specific gene variants called Single Nucleotide Polymorphisms (or SNPs), changes between variants are represented by a sequence of sites, each one bearing a specific value called allele. In almost all cases, for each site at most two different alleles are present in the population, one of which is called major and the other one minor. The sequence of alleles along a chromosome is

\footnotetext{
*Paola Bonizzoni is with DISCo, Univ. Milano-Bicocca

${ }^{\dagger}$ Gianluca Della Vedova is with Dip. Statistica, Univ. Milano-Bicocca

${ }^{\ddagger}$ Riccardo Dondi is with Dip. Scienze dei Linguaggi, della Comunicazione e degli Studi Culturali, Univ. Bergamo

${ }^{\S}$ Yuri Pirola is with DISCo, Univ. Milano-Bicocca

๑ Romeo Rizzi is with DIMI, Univ. Udine
} 
called haplotype, while a genotype is a sequence of unordered pairs of alleles that appear in each site of the two copies of the chromosome. Haplotype data are crucial in genetic population studies. The current technology for finding the two haplotypes of an individual is too expensive to be used in genetic studies of a population. Fortunately it is much cheaper to determine the xor-genotype for each individual, that is the set of sites for which the individual is heterozygous, i.e. bearing both a major and a minor allele. The term xor-genotype derives from the fact that a site is reported in the genotype if and only if the two alleles in the site are different. Thus a xor-genotype lists only heterozygous sites, while excluding sites bearing identical alleles, called homozygous sites.

The problem of reconstructing the haplotypes resolving a given set of xorgenotypes naturally arises and represents an interesting case of the process of inferring haplotypes from general genotypes (phasing).

Polynomial time algorithms for the problem have been developed [2, 3] in the framework of the Perfect Phylogeny model, originally proposed by Gusfield [12] to solve the phasing problem.

In this paper, we investigate the problem under the parsimonious principle that asks for a smallest set of haplotypes resolving all input xorgenotypes: such problem will be called Pure Parsimony Xor HaploTYPING (PPXH).

Let $\Sigma$ be a set of sites (also called characters). Then a xor-genotype (or simply a genotype) $x$ is a non-empty subset of $\Sigma$, and a haplotype $h$ is a (possibly empty) subset of $\Sigma$. Given two distinct haplotypes $h_{1}, h_{2}$, then the pair $\left(h_{1}, h_{2}\right)$ resolves the xor-genotype $x$ iff $x=h_{1} \oplus h_{2}$, where $\oplus$ is defined as the classical symmetric difference of $h_{1}$ and $h_{2}$, i.e. the set of characters that are present in exactly one of $h_{1}$ and $h_{2}$. A set $H$ of haplotypes resolves a set $X$ of xor-genotypes if for each genotype $x \in X$, there exists a pair of haplotypes in $H$ that resolves $x$.

We are now able to formally introduce the problem that we will study in this paper.

Problem 1. Pure Parsimony Xor Haplotyping (PPXH). The instance of the problem is a set $X$ of xor-genotypes, and the goal is to compute a smallest set $H$ of haplotypes resolving $X$.

The pure parsimony model has been used as an approach to the phasing problem over regular genotypes (i.e. where alleles of each homozygous site are specified) [13]. There is a rich literature in this area; in particular the APX-hardness [15] of the problem and the lack of good approximation guarantees have led many researchers to the design of methods based on linear programming techniques to find solutions of the problem [6]. Indeed, the best known approximation algorithm yields approximation guarantees of $2^{k-1}$ where $k$ is the maximum number of heterozygous sites appearing in 
each genotype [15]. Restricted cases of the problem with polynomial time solutions have been studied [21, 16].

In the paper we investigate the PPXH problem mainly by devising exact solutions of the problem by either considering fixed-parameter tractability or polynomial time algorithms for some restricted instances of the problem. We introduce a new graph representation of xor-genotypes and haplotypes, called xor-graph, that is crucial in the study of the PPXH problem. Indeed most of the results that we will present rely on combinatorial properties of xor-graphs.

Initially we will show that the PPXH problem is equivalent to the problem of building a xor-graph with the fewest possible vertices. Afterwards we design two polynomial time solutions for restricted instances of the PPXH problem. Subsequently we design a fixed-parameter algorithm of $O\left(m n+2^{k^{2}} k m\right)$ time complexity, for $k$ the size of the optimum solution. Moreover we provide a $l$-approximation algorithm, where $l$ is the maximum number of occurrences of a character in the set of input genotypes. Finally we propose a heuristic for the general problem and an experimental analysis on real and artificial datasets. The experimental analysis shows that the heuristic is effective on a large class of instances of various sizes where other methods, such as the ILP formulation proposed by Brown and Harrower [6], are not applicable.

\section{Basic Properties}

A fundamental idea used in our paper is a graph representation of a feasible solution. More precisely, given a set $X$ of xor-genotypes, the representation of a set $H$ of haplotypes resolving $X$ is the graph $\mathcal{G}=(H, E)$, called $x$ orgraph associated with $H$, where edges of $\mathcal{G}$ are labeled by a bijective function $\lambda: E \rightarrow X$ such that, for each edge $e=\left(h_{i}, h_{j}\right), \lambda(e)=h_{i} \oplus h_{j}$. The labeling $\lambda$ is generalized to a set $S$ by defining $\lambda(S)=\{\lambda(s) \mid s \in S\}$. We call optimal xor-graph for $X$, a xor-graph associated with an optimal solution for $X$ (that is a xor-graph with the minimum number of vertices).

In this section we state some basic combinatorial properties of xor-graphs that will be used to prove the main results of the paper. Among all possible haplotypes, we identify a distinguished haplotype, called null haplotype and denoted by $h_{0}$, which corresponds to the empty set. Since the operation $\oplus$ is associative and commutative, by a slight abuse of language, given a family $F=\left\{s_{1}, \ldots, s_{n}\right\}$ of subsets of a set $\Sigma$ we denote by $\oplus(F)$ the expression $s_{1} \oplus s_{2} \oplus \cdots \oplus s_{n}$.

The cycles of a xor-graph satisfy the following property.

Lemma 2.1. Let $X$ be a set of xor-genotypes, let $\mathcal{G}$ be a xor-graph associated with a set of haplotypes resolving $X$ and let $C$ be the edge set of a cycle of $\mathcal{G}$. Then $\oplus(\lambda(C))$ is equal to the empty set. 
Proof. By definition of cycle, $C$ consists of a set $\left\{\left(h_{1}, h_{2}\right),\left(h_{2}, h_{3}\right), \ldots,\left(h_{n}, h_{n+1}\right)\right\}$, with $h_{1}=h_{n+1}$. By definition of xor-graph, $\oplus(\lambda(C))=\oplus_{i=1}^{n}\left(h_{i} \oplus h_{i+1}\right)$. By the associativity and commutativity of $\oplus, \oplus(\lambda(C))=\left(h_{1} \oplus h_{n+1}\right) \oplus$ $\left(h_{2} \oplus h_{2}\right) \oplus \ldots \oplus\left(h_{n} \oplus h_{n}\right)$. Since $h_{1}=h_{n+1}$ and $h \oplus h=\varnothing$ for each $h$, we obtain $\oplus(\lambda(C))=\varnothing$.

The above property of cycles of a xor-graph is sufficient to construct a set of haplotypes resolving a set of genotypes from a xor-graph. Let $X$ be an instance of PPXH and let $\mathcal{G}=(V, E)$ be a graph whose edges are biunivocally labeled by a function $\lambda: E \rightarrow X$ such that $\oplus(\lambda(C))=\varnothing$ for each cycle $C$ of the graph. Then it is immediate to compute a feasible solution $H$ from $\mathcal{G}$ where $|H| \leq|V|$. More precisely, we associate a haplotype with each vertex of $\mathcal{G}$ as follows. Associate the null haplotype $h_{0}$ with any vertex in each connected component of $\mathcal{G}$. Perform a depth-first visit of each connected component of $\mathcal{G}$, starting from the vertex associated with $h_{0}$. When visiting a new vertex $v$ of $\mathcal{G}$ there must exist an edge $e=(v, w)$ so that the haplotype $w_{h}$ has been previously assigned to $w$. Then associate the haplotype $w_{h} \oplus \lambda(e)$ with $v$.

It is not hard to verify that our construction guarantee that $H$ is actually a feasible solution of $X$, that is for each edge $e=(v, w)$ of $\mathcal{G}, v_{h} \oplus w_{h}=\lambda(e)$, where $v_{h}$ and $w_{h}$ are respectively the haplotypes associated with $v$ and $w$. It is trivial to notice that the property holds for all edges that are part of the spanning forest computed by the depth-first search. Therefore we can restrict our attention to edges $e=(v, w)$ that are not in such spanning forest. Since $v$ and $w$ are in the same connected component of $\mathcal{G}$ the spanning tree $T$ of the connected component contains both $v$ and $w$. Let $x$ be the least common ancestor of $v$ and $w$ in $T$. By construction the two paths of $T$, both starting from $x$ and ending one in $v$ and the other in $w$ are edge disjoint. Let us denote by $P_{v}$ and $P_{w}$ respectively the edges of the paths ending in $v$ and $w$, and let $x_{h}$ be the haplotype associated with $x$. Now we want to prove that $v_{h} \oplus w_{h}=\lambda(e)$. It is immediate to verify that $v_{h}=\bigoplus\left(\lambda\left(P_{v}\right)\right) \oplus x_{h}$ and $w_{h}=\bigoplus\left(\lambda\left(P_{w}\right)\right) \oplus x_{h}$. Since the edges in $P_{v} \cup P_{w} \cup\{e\}$ form a simple cycle of $\mathcal{G}$, by Lemma 2.1 we can conclude $\lambda(e)=\bigoplus\left(\lambda\left(P_{v}\right)\right) \oplus \bigoplus\left(\lambda\left(P_{w}\right)\right)$, completing the proof.

The following results justify our attention to connected xor-graphs and their cuts.

Lemma 2.2. Let $X$ be a set of xor-genotypes and let $\mathcal{G}$ be a xor-graph associated with a set $H$ of haplotypes resolving $X$. Let $\alpha$ be any character of $\Sigma$. Then the set $A$ of edges of $\mathcal{G}$ whose label contains $\alpha$ is a cut of $\mathcal{G}$.

Proof. Let $H_{\alpha}$ be the subset of $H$ containing the character $\alpha$, and let $\bar{H}_{\alpha}=$ $H \backslash H_{\alpha}$. Let $E^{\prime}$ be the edges of $\mathcal{G}$ with an endpoint in $H_{\alpha}$ and one in $\bar{H}_{\alpha}$ (clearly $E^{\prime}$ is a cut of $\mathcal{G}$.) Notice that $E^{\prime}$ is exactly the set of edges 
connecting a haplotype containing $\alpha$ and a haplotype not containing $\alpha$, therefore $E^{\prime}=A$.

Lemma 2.3. Let $X$ be a set of xor-genotypes, and let $\mathcal{G}=(H, E)$ be a disconnected xor-graph for $X$. Then $\mathcal{G}$ is not an optimal xor-graph of $X$

Proof. Since $\mathcal{G}$ has at least two connected components $C_{1}$ and $C_{2}$, we denote with $a_{1}, a_{2}$ two vertices from $C_{1}$ and $C_{2}$ respectively. Construct the set $H^{\prime}$ from $H$ by replacing each haplotype $h \in C_{1}$ by $h \oplus a_{1}$ and each haplotype $h \in C_{2}$ by $h \oplus a_{2}$. Since $C_{1}$ and $C_{2}$ are not connected, the set of genotypes resolved by $H^{\prime}$ is equal to that of $H$.

But both $a_{1}$ and $a_{2}$ are replaced by the null haplotype in $H^{\prime}$, therefore $\left|H^{\prime}\right|$ is strictly smaller than $|H|$.

Instances and solutions of the PPXH problem can be represented by binary matrices. More precisely, we can have a genotype matrix associated with a set of xor-genotypes and a haplotype matrix associated with a set of haplotypes. In both matrices each column is uniquely identified by a character in $\Sigma$, while the rows of a genotype matrix (respectively haplotype matrix) correspond to the genotypes (resp. haplotypes).

For example let $\Sigma$ be the set $\{a, b, c, d, e\}$ and let $X$ be the set of xorgenotypes $\{\{a, b\},\{a, b, c\},\{b, c\},\{c, d, e\},\{a\},\{e\},\{a, c, e\}\}$. A possible, albeit suboptimal, set of haplotypes resolving $X$ is $\{\varnothing,\{c, d\},\{a, b, c, d\},\{a, c\},\{a, d\},\{d\},\{e\}\}$. The matricial representation of both sets is in Table 1, while the associated xor-graph is represented in Figure 1 .

Table 1: Example of genotype (left) and haplotype (right) matrices.

\begin{tabular}{l|lllll} 
& $a$ & $b$ & $c$ & $d$ & $e$ \\
\hline$g_{1}$ & 1 & 1 & 0 & 0 & 0 \\
$g_{2}$ & 1 & 1 & 1 & 0 & 0 \\
$g_{3}$ & 0 & 1 & 1 & 0 & 0 \\
$g_{4}$ & 0 & 0 & 1 & 1 & 1 \\
$g_{5}$ & 1 & 0 & 0 & 0 & 0 \\
$g_{6}$ & 0 & 0 & 0 & 0 & 1 \\
$g_{7}$ & 1 & 0 & 1 & 0 & 1
\end{tabular}

\begin{tabular}{l|lllll} 
& $a$ & $b$ & $c$ & $d$ & $e$ \\
\hline$h_{1}$ & 0 & 0 & 0 & 0 & 0 \\
$h_{2}$ & 0 & 0 & 1 & 1 & 0 \\
$h_{3}$ & 1 & 1 & 1 & 1 & 0 \\
$h_{4}$ & 1 & 0 & 1 & 0 & 0 \\
$h_{5}$ & 1 & 0 & 0 & 1 & 0 \\
$h_{6}$ & 0 & 0 & 0 & 1 & 0 \\
$h_{7}$ & 0 & 0 & 0 & 0 & 1
\end{tabular}

Given an ordering of the character set (that is $\Sigma=\left\langle\sigma_{1}, \ldots, \sigma_{|\Sigma|}\right\rangle$ ), the entry in the $i$-th row and $j$-th column of a genotype matrix (respectively, haplotype matrix) is 1 if $\sigma_{j}$ belongs to the $i$-th genotype (respectively, $i$ th haplotype) and is equal to 0 otherwise. In the following we identify rows of a genotype (or haplotype) matrix with the corresponding genotypes (or haplotypes). Given a matrix $M$, we denote by $M[\cdot, A]$ (by $M[B, \cdot]$, respectively) the submatrix of $M$ induced by the set $A$ of columns (by the set $B$ of rows, respectively). 


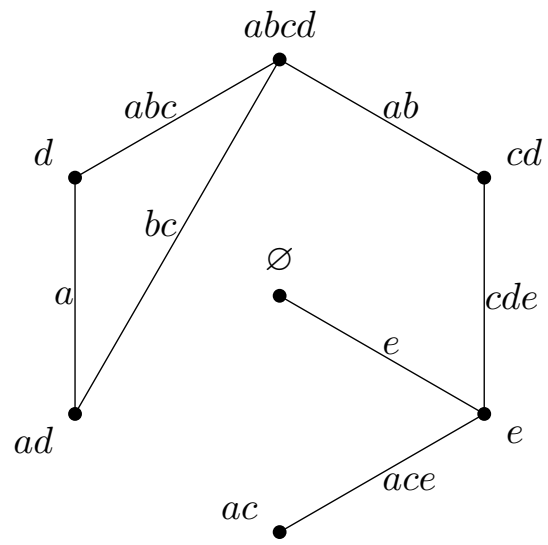

Figure 1: Xor-graph representing the set of haplotypes in Table 1.

Given a genotype or haplotype matrix $M$ over $\Sigma$, we will say that a subset $\Sigma_{1}$ of $\Sigma$ is a linearly dependent set of characters (or, simply, a dependent set of characters) in matrix $M$ if there exists a non-empty subset $\Sigma_{2}$ of $\Sigma_{1}$ such that, for each row $i, \oplus_{\sigma \in \Sigma_{2}} M[i, \sigma]=0$. Otherwise it is called linearly independent (or simply independent).

While solving the PPXH problem, we can restrict our attention to a maximal independent subset of characters as stated in the following lemma.

Lemma 2.4. Let $X$ be a xor-genotype matrix and $H$ be a haplotype matrix over the same character set $\Sigma$. Let $\Sigma_{1}$ be a maximal independent subset of $\Sigma$ in $X$. Then, $H$ resolves $X$ if and only if $H\left[\cdot, \Sigma_{1}\right]$ resolves $X\left[\cdot, \Sigma_{1}\right]$

Proof. The only-if part is obviously true because $H\left[\cdot, \Sigma_{1}\right]$ and $X\left[\cdot, \Sigma_{1}\right]$ are two submatrices of $H$ and $X$ respectively. The if part can be proved by constructing a feasible solution $H$ for $X$ from the smaller solution $H\left[\cdot, \Sigma_{1}\right]$ for $X\left[\cdot, \Sigma_{1}\right]$ (for simplicity we will refer to the two submatrices respectively as $H^{\prime}$ and $X^{\prime}$ ). For each character $\alpha \in \Sigma \backslash \Sigma_{1}$, since $\Sigma_{1} \cup\{\alpha\}$ is dependent there exists a non-empty subset $\Sigma_{\alpha}$ of $\Sigma_{1}$ such that, for each genotype $x$, $X[x, \alpha]=\oplus_{\sigma \in \Sigma_{\alpha}} X^{\prime}[x, \sigma]$. Set the entry $H[x, \alpha]$ to $\oplus_{\sigma \in \Sigma_{\alpha}} H^{\prime}[x, \sigma]$.

We claim that $H$ resolves $X$. Since $H^{\prime}$ resolves $X^{\prime}$, it suffices to prove that for each character $\alpha \in \Sigma \backslash \Sigma_{1}, H\left[h_{1}, \alpha\right] \oplus H\left[h_{2}, \alpha\right]=X[x, \alpha]$, for some pair of haplotypes $h_{1}, h_{2}$. We already know that for each genotype $x^{\prime}$ of $X^{\prime}$, there is a pair $\left(h_{1}^{\prime}, h_{2}^{\prime}\right)$ of haplotypes in $H^{\prime}$ that resolves $x^{\prime}$. Notice that $X[x, \alpha]=\oplus_{\sigma \in \Sigma_{\alpha}} X^{\prime}[x, \sigma]$ since $\Sigma_{1}$ is a maximal subset of independent characters of $X$. Since $H^{\prime}$ resolves $X^{\prime}, \oplus_{\sigma \in \Sigma_{\alpha}} X^{\prime}[x, \sigma]=\oplus_{\sigma \in \Sigma_{\alpha}}\left(H^{\prime}\left[h_{1}, \sigma\right] \oplus\right.$ $\left.H^{\prime}\left[h_{2}, \sigma\right]\right)$. Moreover, by the associativity of $\oplus, \oplus_{\sigma \in \Sigma_{\alpha}}\left(H^{\prime}\left[h_{1}, \sigma\right] \oplus H^{\prime}\left[h_{2}, \sigma\right]\right)=$ $\left(\oplus_{\sigma \in \Sigma_{\alpha}} H^{\prime}\left[h_{1}, \sigma\right]\right) \oplus\left(\oplus_{\sigma \in \Sigma_{\alpha}} H^{\prime}\left[h_{2}, \sigma\right]\right)$. Finally, by our construction of the columns of $H$ corresponding to characters in $\Sigma \backslash \Sigma_{1},\left(\oplus_{\sigma \in \Sigma_{\alpha}} H^{\prime}\left[h_{1}, \sigma\right]\right) \oplus$ $\left(\oplus_{\sigma \in \Sigma_{\alpha}} H^{\prime}\left[h_{2}, \sigma\right]\right)=H\left[h_{1}, \alpha\right] \oplus H\left[h_{2}, \alpha\right]$, hence completing the proof.

Notice that, given a $n \times m$ xor-genotype matrix $X$, a maximal subset 
of independent characters in $X$ can be extracted by applying the Gausselimination algorithm on the matrix $X$ in $O\left(n m^{2}\right)$ time. Observe that the proof of Lemma 2.4 is constructive and shows how to compute efficiently a solution $H$ for $X$ from a solution $H\left[\cdot, \Sigma_{1}\right]$ for $X\left[\cdot, \Sigma_{1}\right]$. We can introduce another simplification of the instance which can be performed efficiently. It affects the construction of the xor-graph and allows an efficient reconstruction of an optimal xor-graph for the general instance, given a xor-graph for the reduced or simplified instance.

Lemma 2.5. Let $X$ be an instance of PPXH, and let $\alpha$ be a character of $X$ such that there exists exactly one genotype $x \in X$ with $\alpha \in x$. Then there exists an optimal xor-graph $\mathcal{G}$ for $X$ such that there is a vertex $v$ of $\mathcal{G}$ with exactly one edge $e$ incident on $v$ and $\lambda(e)=x$.

Proof. Let $\mathcal{G}$ be an optimal xor-graph $\mathcal{G}$ for $X$. Since $\alpha$ appears in only one genotype in $X$, there is exactly one edge $e$ of $\mathcal{G}$ such that $\alpha \in \lambda(e)$. By Lemma 2.2 removing $e$ from $\mathcal{G}$ results in a bipartition $\left\{H_{\alpha}, \bar{H}_{\alpha}\right\}$ where $H_{\alpha}$ consists of the haplotypes containing $\alpha$. Let $v \in H_{\alpha}$ and $w \in \bar{H}_{\alpha}$ be the two endpoints of $e$, and let $D$ be the set of vertices of $H_{\alpha}$ adjacent to $v$. Change each haplotype in $h \in H_{\alpha} \backslash\{v\}$ to $h \oplus v \oplus w$, obtaining a new xor-graph $\mathcal{G}_{1}$.

By construction, $\mathcal{G}_{1}$ has set of edges $E_{1}=E \backslash\{(v, d) \mid d \in D\} \cup\{(w, d) \mid$ $d \in D\}$. Indeed, let $e=(v, d)$ be any edge of $\mathcal{G}$ connecting $v$ with a vertex $d \in D$; in $\mathcal{G}_{1}$ there is an edge $f=(w, d)$ such that $\lambda(e)=\lambda(f)$. It is immediate to notice that $\mathcal{G}$ and $\mathcal{G}_{1}$ have the same number of vertices, therefore $\mathcal{G}_{1}$ is optimal and satisfies the statement of the lemma.

Also the proof of Lemma 2.5 is constructive and can be exploited directly in an algorithm to simplify the instance of the problem. More precisely, the removal of a genotype and a character as stated by Lemma 2.5 can be repeated until all characters appear in at least two genotypes (or we obtain the special case of an instance containing only one genotype; in such case the optimal solution is trivially made by two haplotypes.) Following the same idea, if the set of characters is linearly dependent, we can extract a maximal subset of linearly independent characters. Moreover the executions of the two reductions can be intertwined until none of those reductions can be performed.

An instance $X$ of PPXH is called reduced if (i) $X$ consists of only one genotype, or the two following conditions are satisfied: (ii a) the set of characters of $X$ are independent and (ii b) each character appears in at least two genotypes. Lemmas 2.4 and 2.5 justify the fact that we will assume in the rest of the paper that all instances are reduced, as the reduction process can be performed efficiently, and we can easily compute a solution of the original instance given a solution of a reduced instance (see Algorithm 1 for a more detailed description of the reduction process). 


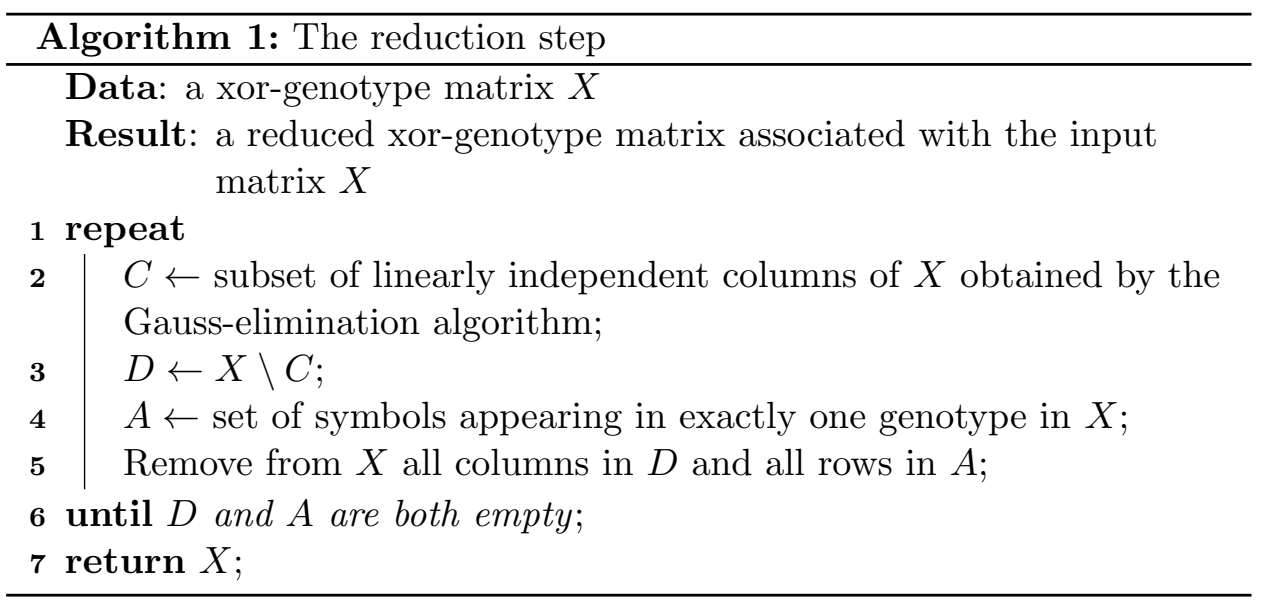

The reduction process leads us to an important lower bound on the size of the optimum.

Lemma 2.6. Let $X$ be a reduced genotype matrix having $n$ rows and $m$ columns. Then any haplotype matrix $H$ resolving $X$ has at least $m+1$ rows.

Proof. Let $\mathcal{G}$ be a xor-graph for $X$. By Lemma 2.2, each character $\alpha$ induces a cut in graph $\mathcal{G}$. Each cut can be represented as $n$-bit binary vector $c_{\alpha}$ in which each element $c_{\alpha}[i]$ is equal to 1 if and only if the genotype $x_{i}$ belongs to the cut. Clearly, such vector is precisely the column vector corresponding to character $\alpha$ of matrix $X$. Thus, since the characters are independent, also the family of the cuts (represented as binary vectors) induced by the set of characters is linearly independent. By Theorem 1.9.6 of [7] all connected graphs with $m$ independent cuts have at least $m+1$ vertices.

As a consequence of Lemma 2.6, in a reduced xor-genotype matrix, the number of rows is greater than or equal to the number of columns. In fact, in any matrix, the number of linearly independent columns is equal to the number of linearly independent rows and, clearly, is bounded by the minimum between the number of columns and the number of rows.

The process of reducing a xor-genotype matrix by restricting ourselves to a maximal subset of independent characters is an application of the kernelization technique for designing a fixed-parameter algorithm [8]. The technique consists of reducing the original instance to a new instance whose size depends only on the parameter (in our case the size of the optimal solution.) The size of the reduced xor-genotype matrix is clearly bounded by a polynomial function of the optimum $k$ since at most $O\left(k^{2}\right)$ distinct genotypes can be generated by $k$ distinct haplotypes and, by the previous consideration, the number of columns is less than the number of rows. As a result, the number of entries of a reduced xor-genotype matrix is bounded by $O\left(k^{4}\right)$. 


\section{Algorithms for Restricted Instances}

In this section we investigate two restrictions of the PPXH problem obtained by bounding the number of characters that can appear in each genotype and the number of genotypes where a character can occur. Those restrictions are summarized by the following formulation.

Problem 2. Constrained Pure Parsimony Xor Haplotyping (PPXH $(p, q))$. The instance consists of a set $X$ of xor-genotypes, where each xor-genotype $x \in X$ contains at most $p$ characters, and each character appears in at most $q$ xor-genotypes. The goal is to compute a minimum cardinality set $H$ of haplotypes that resolves $X$. We use the symbol $\infty$ when one of parameters $p$ or $q$ is unbounded.

More precisely we will present efficient algorithms for the case when each character is contained in at most two xor-genotypes $(\operatorname{PPXH}(\infty, 2))$ and the case that each genotype consists of at most two characters $(\operatorname{PPXH}(2, \infty))$.

\subsection{A Polynomial Time Algorithm for $\operatorname{PPXH}(\infty, 2)$}

The structure of the cycles in a xor-graph characterizes the solutions for the $\operatorname{PPXH}(\infty, 2)$ problem as stated in the following Lemma.

Lemma 3.1. Let $X$ be a reduced instance of $P P X H(\infty, 2)$, let $\mathcal{G}$ be an optimal xor-graph for $X$, and let $e$ be an edge of $\mathcal{G}$. Then e belongs to exactly one simple cycle of $\mathcal{G}$.

Proof. Assume to the contrary that an edge $e$ belongs to two cycles $C_{1}$ and $C_{2}$. Notice that the three sets $C_{1} \backslash C_{2}, C_{2} \backslash C_{1}, C_{1} \cap C_{2}$ are pairwise disjoint and not empty. Let $d$ be any element of $\lambda\left(C_{1} \cap C_{2}\right)$. By Lemma 2.1. $\oplus\left(\lambda\left(C_{1} \backslash C_{2}\right)\right)=\oplus\left(\lambda\left(C_{2} \backslash C_{1}\right)\right)=\oplus\left(\lambda\left(C_{1} \cap C_{2}\right)\right)$. Consequently there exist three distinct edges $e_{1} \in C_{1} \backslash C_{2}, e_{2} \in C_{2} \backslash C_{1}, e_{3} \in C_{1} \cap C_{2}$ such that $\lambda\left(e_{1}\right)$, $\lambda\left(e_{2}\right), \lambda\left(e_{3}\right)$ all contain $d$, which contradicts the fact that there are only two genotypes containing $d$. By the first part of the proof, we have now to prove that $e$ belongs to at least a cycle of $\mathcal{G}$.

Assume to the contrary that $X$ is a smallest counterexample, that is no such xor-graph exists for $X$, while such graph exists for all reduced instances with fewer genotypes, and let $\mathcal{G}$ be any optimal xor-graph for $X$. Since there is an edge that does not belong to any cycle of $\mathcal{G}$, there is a character $a$ such that both edges $e_{1}$ and $e_{2}$ containing $a$ do not belong to any cycle. Notice that two such edges must exists, since the instance is reduced. Let us denote by $\alpha, \beta$ and $\gamma$ respectively the sets $\lambda\left(e_{1}\right) \cap \lambda\left(e_{2}\right), \lambda\left(e_{1}\right) \backslash \lambda\left(e_{2}\right), \lambda\left(e_{2}\right) \backslash \lambda\left(e_{1}\right)$.

Compute a new reduced instance $X_{1}$ from $X$ by removing the xorgenotypes $e_{1}$ and $e_{2}$, and adding a new genotype $x_{c}=\beta \cup \gamma=e_{1} \oplus e_{2}$. Clearly $X_{1}$ is a reduced instance of $\operatorname{PPXH}(\infty, 2)$ smaller than $X$, therefore $X_{1}$ admits an optimal graph $\mathcal{G}_{1}$ where all edges are in some cycle. Let 
us consider the unique cycle $C$ of $\mathcal{G}_{1}$ containing the edge $e=(u, v)$, with $\lambda(e)=x_{c}$. Now, starting from $\mathcal{G}_{1}$, compute a xor-graph $\mathcal{G}_{2}$ for instance $X$, by adding to $\mathcal{G}_{1}$ a new vertex $w$, two edges $e_{1}^{\prime}=(u, w), e_{2}^{\prime}=(w, v)$, so that $\lambda\left(e_{1}^{\prime}\right)=e_{1}$ and $\lambda\left(e_{2}^{\prime}\right)=e_{2}$, and by removing edge $e$. The graph $\mathcal{G}_{2}$ is a xor-graph of $X$ as $x_{c}=e_{1} \oplus e_{2}$. Clearly the newly obtained graph $\mathcal{G}_{2}$ is a xor-graph for $X$ satisfying the requirements of the lemma and $\mathcal{G}_{2}$ contains one more vertex than $\mathcal{G}_{1}$.

We have to prove that $\mathcal{G}_{2}$ is optimal, therefore assume that $\mathcal{G}_{2}$ is not optimal and let $\mathcal{G}_{*}$ be an optimal xor-graph for $X$, that is $\mathcal{G}_{*}$ has no more vertices than $\mathcal{G}_{1}$. It is immediate to notice that contracting each of $e_{1}$ and $e_{2}$ into single vertices result in a xor-graph that is a solution of $X_{1}$ with fewer vertices than $\mathcal{G}_{1}$, hence violating the optimality of $\mathcal{G}_{1}$.

Since the optimal xor-graph is connected (Lemma 2.3) and consists of a set of edge-disjoint cycles (Lemma 3.1), the size of the optimum solution is equal to $|X|+1-|\mathcal{C}|$, for $|X|$ the number of genotypes or edges of the graph and $|\mathcal{C}|$ the number of simple cycles of the graph, since any set of $|\mathcal{C}|$ simple cycles on a graph with at most $|X|-|\mathcal{C}|$ must share at least an edge.

Algorithm 2 solves the $\operatorname{PPXH}(\infty, 2)$ problem by computing the set $\mathcal{C}$ of all simple cycles of an optimal xor-graph. In fact Lemma 3.1 allows us to introduce a binary relation $R$ between genotypes, where two genotypes are related if and only if they share a common character. By Lemma 3.1 any two genotypes (or edges of the xor-graph) that are related must also belong to the same simple cycle. It is immediate to notice that the partition of the edges of the xor-graph into simple cycles is equal to the most refined partition of edges such that any two edges sharing a common character belong to the same set of such partition. In fact Algorithm 2 computes exactly the closure of $R$.

\subsection{A Polynomial Time Algorithm for $\operatorname{PPXH}(2, \infty)$}

For simplicity's sake we will assume that the instance of the problem is a genotype matrix $X$ and the desired output is a haplotype matrix $H$.

We remember that in both matrices the columns are indexed by characters therefore we will denote by $X[\cdot, \sigma]$ (respectively $H[\cdot, \sigma]$ ) the column of $X$ (resp. $H$ ) indexed by the character $\sigma$. The algorithm is based on Lemmas 2.4 and 2.6 .

In fact we will first compute a largest set $\Sigma_{1}$ of independent characters in $X$. Moreover for each character $\alpha \in \Sigma \backslash \Sigma_{1}$ we determine the subset $\Sigma_{\alpha}$ of $\Sigma_{1}$ such that $X[\cdot, \alpha]=\oplus_{\sigma \in \Sigma_{\alpha}} X[\cdot, \sigma]$. Notice that this step can be carried over by a simple application of the Gauss-elimination algorithm.

Let $X^{\prime}$ be the submatrix $X\left[\cdot, \Sigma_{1}\right]$. An optimal solution of the instance $X^{\prime}$ is the matrix $H^{\prime}$ containing $\left|\Sigma_{1}\right|+1$ rows. More precisely the $i$-th row of 


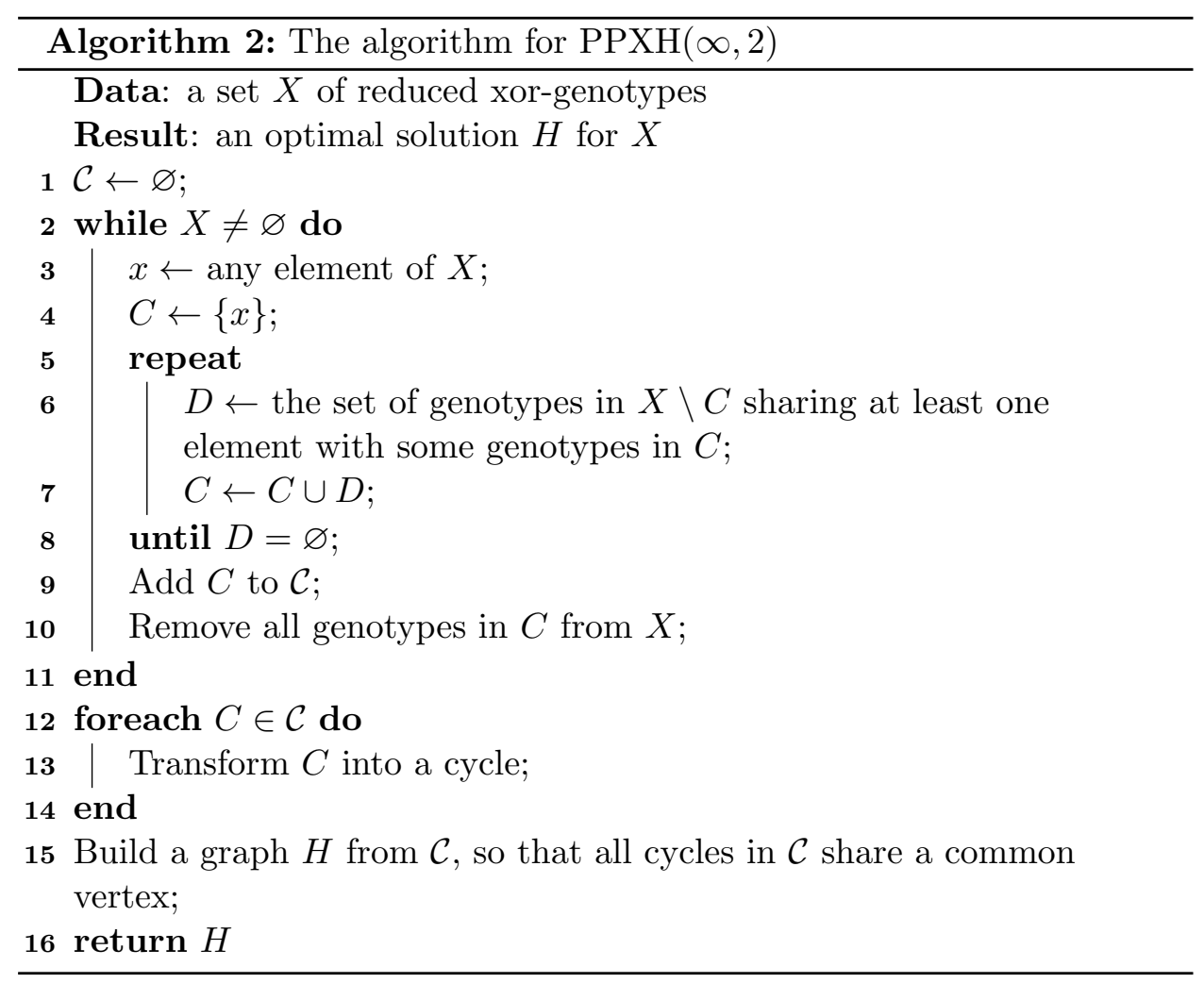

$H^{\prime}$, for $1 \leq i \leq\left|\Sigma_{1}\right|$, consists of all zeroes, except for the $i$-th column (where it contains 1 ). The last row contains only zeroes.

Clearly $H^{\prime}$ resolves $X^{\prime}$. In fact it is immediate to notice that each row of $X^{\prime}$ contains at most two 1s, as the same property holds for $X$, therefore for each row $r$ of $X^{\prime}$ there are two rows of $H^{\prime}$ resolving $r$. The optimality of such solution is a direct consequence of Lemma 2.6. Clearly $H^{\prime}$ is not a feasible solution of the original instance $X$, but such a feasible solution $H$ can be easily computed from $H^{\prime}$ by adding, for each character $\alpha \in \Sigma \backslash \Sigma_{1}$, a column equal to $\oplus_{\sigma \in \Sigma_{\alpha}} H^{\prime}[\cdot, \sigma]$ (where $\Sigma_{\alpha}$ satisfies $X[x, \alpha]=\oplus_{\sigma \in \Sigma_{\alpha}} X^{\prime}[x, \sigma]$ for each genotype $x$.) The matrix $H$ is a feasible solution as shown in the proof of Lemma 2.4 .

\section{Fixed-Parameter Tractability of PPXH}

As observed at the end of Section 2, the reduction of an instance of the unrestricted PPXH problem lead us to a fixed-parameter algorithm (where the parameter is the optimum). Moreover we can observe that there exists another fixed-parameter algorithm for the unrestricted PPXH problem without using the reduction of input instance. Let $H$ be a set of haplotypes and let $X$ be a set of genotypes resolved by $H$; in the following we will de- 
note $|X|$ by $n$. Since $H$ can resolve at most $\left(\begin{array}{c}|H| \\ 2\end{array}\right)$ genotypes, $n \geq|H| \geq \sqrt{2 n}$. In other words, if $k$ is the size of the minimum-cardinality set of haplotypes resolving $X, n \in O\left(k^{2}\right)$.

The number of the possible graphs with at most $n+1$ vertices and exactly $n$ edges is no more than $2^{2 n \log _{2}(n+1)}=(n+1)^{2 n}$ which, by our previous observation, is $O\left(k^{4 k^{2}}\right)$, i.e. a function dependent only on $k$. The time needed to check if one of such graphs is a xor-graph for $H$ is clearly polynomial in $n$ and thus we can immediately derive a fixed-parameter algorithm to find an optimal xor-graph for $X$.

The time complexity of the algorithm is well beyond what is deemed acceptable in practice, therefore we propose a more efficient algorithm that is based on the matrix representation of genotypes and haplotypes.

In the following we will assume that the genotype matrix $X$ is reduced, and that $X$ has $n$ rows and $m$ independent columns, and that we are looking for a haplotype matrix $H$ with at most $k$ distinct rows that resolves $X$. The basic idea of our algorithm is to enumerate all possible haplotype matrices.

In the naïve approach, testing if a haplotype matrix resolves a given genotype matrix requires $O\left(k^{2} n m\right)$ time because each pair of haplotypes has to be considered and then each resulting genotype has to be searched in the genotype matrix. Our strategy, instead, is to enumerate all the haplotype matrices by changing only one haplotype each time, in such a way that only $k-1$ new pairs of haplotypes must be considered when testing if $H$ resolves the set $X$.

We use Gray codes [18 to visit all the haplotype matrices in such a way that each pair of consecutive matrices differs by a single bit and, thus, by a single haplotype. More precisely, we enumerate all $k \times m$ matrices by generating all $\mathrm{km}$-long bit vectors. Indeed, the bits from position $(i-1) \mathrm{m}+1$ to position $i m$ in a $\mathrm{km}$-long vector give the $i$-th row of the matrix (for $1 \leq i \leq k)$. The fastest known algorithm for computing the next vector of a Gray code requires constant time for each invocation [4].

Observe that the naïve algorithm requires $O(n m)$ time to test if there is a genotype in matrix $X$ resolved by a pair of haplotypes. By representing the set of the row vectors of matrix $X$ as a binary trie [9], the time required to get the index of the row containing a $m$-long binary vector is reduced to $O(m)$.

The details of the fixed-parameter algorithm are given in the Algorithm 3, where we also use some additional data structures: the array $R e$ solvedByHowMany which associates with each genotype the number of pairs of haplotypes resolving such genotype, and ListResolvedGenotypes which associates with each haplotype $h$ a list of the relevant pairs of haplotypes in which $h$ is involved. In fact, the elements of the lists in ListResolvedGenotypes are triples $\left(h_{1}, h_{2}, x\right)$ where $\left(h_{1}, h_{2}\right)$ is a pair of haplotypes resolving $x$.

Notice that the outermost foreach loop (lines 6-25) iterates $2^{k m}$ times, 
while the for loop at lines 1521 iterates $k$ times. Each iteration of the latter loop consists of a lookup in a trie (which can be done in $O(m)$ time) and updating in constant time some arrays and lists. Since each list can contain at most $k$ elements, the time required for each iteration of the outermost loop is $O(k m)$, resulting in an overall $O\left(n m+2^{k^{2}} k m\right)$ time complexity.

\section{An Approximation Algorithm}

We present a simple approximation algorithm, detailed as Algorithm 4 , which guarantees for a reduced instance $X$ of PPXH an approximation factor $l$, where $k$ is the maximum number of xor-genotypes where each character appears.

Initially the set $H$ of haplotypes computed by the algorithm contains only the null haplotype. While the set of genotypes is not empty, pick a character $\alpha$ that appears in at least a genotype, move to $H$ all genotypes containing $\alpha$, and remove from $X$ all genotypes that are solved by a pair of haplotypes in $H$. Clearly the final set of haplotypes $H$ solves the set of genotypes $X$.

The proposed algorithm returns a solution of size at most $l$ times larger than the optimum which, by Lemma 2.6 , is at least $|\Sigma|+1$. Our algorithm starts with a partial solution $H$ containing only the null haplotype, and at each iteration adds at most $l$ haplotypes to the solution $H$, as $l$ is the maximum number of genotypes containing any character. Since there can be at most $|\Sigma|$ steps, $|H| \leq l|\Sigma|+1$.

Clearly the approximation ratio is at most $(l|\Sigma|+1) /(|\Sigma|+1) \leq l$, completing the proof.

\section{Solving PPXH by a Heuristic Method}

In this section we propose a heuristic algorithm to build a near optimal xor-graph for an input matrix $X$ of genotypes. Observe that an optimal xor-graph for $X$ is a graph having the minimum-cardinality vertex set and where each edge is uniquely labeled by a genotype $X$. By Lemma 2.1, a cycle of the xor-graph consists of a subset $X^{\prime}$ of the input genotypes such that $\oplus X^{\prime}=\varnothing$. Consequently we will call a subset $X^{\prime}$ with $\oplus X^{\prime}=\varnothing$ a candidate cycle.

The basic idea that guides our heuristic is first to select a subset of the candidate cycles of $X$ and then to build a labeled graph (a xor-graph) where the selected candidate cycles are actual cycles. The procedure successively iterates over the genotypes that are not yet successfully realized in the xorgraph.

A related problem is the one called Graph Realization (GR) [20], which consists of building a graph given its fundamental cycles. We recall that the 
set $\mathcal{C}$ of fundamental cycles of a graph $G$ with respect to a fixed spanning tree $T$ of $G$, is defined as $\mathcal{C}=\{$ the unique cycle of $T \cup\{e\} \mid e \in E(G) \backslash E(T)\}$ (see e.g. [7], pag. 26). More precisely, the Graph Realization problem can be formally stated as follows [20]. Given two disjoint sets $T$ and $C$, the input of the GR problem is a family $F$ of subsets of $T \cup C$ such that (i) for each set $F_{i}$ of the family $F, F_{i} \cap C=\left\{c_{i}\right\}$, and (ii) for each pair of subset $F_{i}$ and $F_{j}$ of $F, F_{i} \cap F_{j} \cap C=\varnothing$. The GR problem consists of finding a labeled graph $G=(V, E)$ (if such a graph exists) which realizes $F$, that is there is a bijection between the set $T$ and a spanning tree of $G$, and the elements of each set $F_{i}$ label exactly the edge set of a (simple) cycle of $G$.

In the case that we have selected a set of candidate cycles which are fundamental cycles of a graph $G$, an immediate application of any algorithm solving the GR problem (two almost linear time algorithms exist [5, 10]), gives a xor-graph resolving all those candidate cycles. We have been inspired by those algorithm for GR to develop our heuristic. We denote by $G(F)$ a graph realization $G$ of a family of sets $F$.

The heuristic procedure transforms a $r \times c$ genotype matrix $X$ into an instance of GR as described in the following two main steps. In the first step, the set $T$ is defined as a maximal subset $T=\left\{x_{j_{1}}, \ldots, x_{j_{c}}\right\}$ of linearly independent input genotypes of $X$. This means that any other input genotype $x_{i}$ can be expressed as a linear combination $\alpha_{i, 1} x_{j_{1}} \oplus \ldots \oplus \alpha_{i, c} x_{j_{c}}$ of the genotypes in $T$. Then $C=\left\{c_{1}, \ldots c_{r-c}\right\}$ is defined as consisting of the set of genotypes not in $T$. In a second step, the family of subsets of $T \cup C$ giving an instance of the GR is built by building sets $F_{i}$ such that $F_{i}=\left\{c_{i}\right\} \cup\left\{x_{j_{l}} \in T \mid \alpha_{i, l}=1\right\}$. Informally, $F_{i}$ consists of $c_{i}$ and the unique set $P_{i} \subseteq T$, such that $\oplus P_{i}=\left\{c_{i}\right\}$. An immediate consequence of our definition is that $\oplus F_{i}=\varnothing$, therefore $F_{i}$ is, by definition, a candidate cycle.

Computing the set $T$ from $X$ is simply a matter of running the Gauss elimination algorithm on $X^{T}$ (that is the transpose matrix of $X$.) The family $F$ can be easily inferred by computing the coefficients $\alpha_{i, 1}, \ldots, \alpha_{i, c}$ for all $c_{i} \in C$, where the unknowns $\alpha_{1}^{i} \ldots \alpha_{r}^{i}$ are the coefficients of the linear combination and the binary matrix $M$ is a matrix whose columns are the xor-genotypes in $I$.

Clearly, the Gauss-elimination procedure applied on the matrix $X^{T}$ results in a matrix $R$ whose first $r$ columns form the identity matrix while the other columns are the vectors of the linear combination coefficients.

We have a final hurdle, that is to handle the case where the GR does not exist for the family $F$. Once the family $F$ is identified, the heuristics computes a maximal subfamily $F^{\prime}$ of $F$, so that there exists a GR from $F^{\prime}$. Now, let us detail the construction of the family $F$ giving an instance of GR. The heuristic starts defining $F^{\prime}$ as an empty family and iteratively adding to $F^{\prime}$ a candidate cycle $F_{i}$ if and only if the resulting family admits a Graph Realization. Clearly, this approach ends with a maximal subset of candidate cycles that admits a Graph Realization. The two steps of the 
heuristic procedure are then recursively iterated on the set of xor-genotypes of $X$ that do not label an edge of the computed Graph Realization. The details of the procedure are presented in Algorithm 5 .

Let $n$ and $m$ be, respectively, the number of xor-genotypes and sites. The time complexity of the heuristic is determined by the time complexity of the Gauss elimination algorithm, which requires $O\left(n^{2} m\right)$ time because it is called on matrix $X^{T}$, and of the Graph Realization algorithm, whose best time complexity is $O(\alpha(n, m) n m)$, where $\alpha$ is the inverse Ackermann function. Notice that the Graph Realization algorithm is repeated at most $n$ times in order to compute a maximal subfamily $F^{\prime}$, hence $O\left(\alpha(n, m) n^{2} m\right)$ times. Finally, there is at least one xor-genotype of $X$ that labels an edge of the Graph Realization, hence the total number of iterations is at most $n$, leading to an overall time complexity $O\left(\alpha(n, m) n^{3} m\right)$.

\subsection{Experimental Results}

We have implemented our heuristic as a $\mathrm{C}$ program using the software GREAL [1] as a routine to solve the Graph Realization problem. The GREAL program implements the algorithm of Gavril and Tamari [11] even if its time complexity is $O\left(n m^{2}\right)$ (opposed to the $O(\alpha(n, m) n m$ ) time complexity of the best known algorithm), since it is still effective for our purposes.

The experimental analysis of our heuristic is composed of two parts. In the first part we have applied the algorithm on synthetic instances to evaluate the quality of the results in terms of cardinality of the solutions and running time. In the second part we have assessed the applicability of the heuristic to some real-world large instances.

\subsubsection{Synthetic Data}

Each synthetic instance has been created starting from a set of initial haplotypes and then each xor-genotype has been generated as combination of two haplotypes randomly selected from the initial set. Notice that such process does not guarantee that every haplotype is selected to form a genotype.

We have used two different methods to generate the set of initial haplotypes: (a) pure random generation, and (b) generation under the neutral model. The first strategy, pure random generation, selects uniformly sets of $h$ distinct haplotypes from the set of all binary haplotypes of length $m$. The second strategy, generation under the neutral model, uses the standard Hudson's simulator ms [14] to generate a sample of $h$ haplotypes assuming the neutral model of genetic variation. In this case, the sample of haplotypes can contain repeated elements. Using two different methods to generate the set of initial haplotypes allows us to verify if the behavior of the heuristic is influenced by the choice of the initial haplotypes.

The evaluation criteria, in both cases, were (a) the number of distinct 
haplotypes computed by our method, and (b) its running time. In particular, we have considered as main indicator of the quality of the solutions the ratio $(r)$ between the number of distinct haplotypes of the computed solution and the number of distinct initial haplotypes selected to generate a genotype of the instance. We notice that $r$ is only a proxy for the actual approximation ratio (that is the ratio between the number of distinct computed haplotypes and the size of a optimal solution) achieved by the algorithm, as the number of the selected haplotypes represents only an upper bound of the optimum, thus the ratio $r$ might be strictly less than 1 .

Since the outcome of our heuristic can be influenced by the order of the input genotypes, for each instance we have run the algorithms on ten random permutations of the genotypes, and we have retained only the smallest set of computed haplotypes. The running time refers to the total time required by the heuristic on the 10 permutations of genotypes and has been measured on a standard PC with 1GB of memory with CentOS Linux 5.

The pure random generation strategy is characterized by three parameters, namely the number of input genotypes $(n)$, the number of haplotypes $(h)$, and the number of characters $(m)$. We have considered 4 different values of the parameter $n(100,200,300,400)$, and we have computed the values of $h$ and $m$ from $n$ : in fact those values are $n / 4, n / 3$, and $2 n / 3$. The maximum size of the test instances (400 genotypes and 233 characters) has been chosen in such a way that repeated tests on several instances of the same size would be feasible on a normal computer. In fact, as discussed below, on average the heuristic required roughly an hour on the largest instances, therefore any further increase of the instance size would have made the experimentation impractical.

Table 2 reports the average size of the solutions computed by our heuristic, its average running time, and the average ratio $r$ on 10 random instances generated for each choice of the parameters $n, h$, and $m$.

The second strategy, generation under the neutral model, is characterized by the three parameters $n, m$, and $\rho$, where $n$ is the number of genotypes, $m$ is the number of characters, and $\rho$ is the crossover (or recombination) rate of the Hudson's program. The size of the initial sample of haplotypes has been set equal to the number $n$ of genotypes. Since the sample can contain several copies of the same haplotype, the number of distinct haplotypes randomly selected to form a genotype has been significantly lower than the number of genotypes for almost all of the generated instances.

We considered 30 instances for each choice of the parameters $(n, m, \rho)$ with $n \in\{50,75,100\}, m \in\{50,75,100\}$, and $\rho \in\{0,8,16,24\}$. As for the previous dataset, Table 3 reports the average size of the solution computed by our heuristic, its average running time, and the average ratio $r$.

On both datasets the heuristic produces comparable results. In particular, the average ratio is never larger than 1.57 , while quite often it is close to 1 . In other words, it can often reconstruct a solution of size similar to the 
number of the haplotypes used to generate the instance and, in the worst case, the computed solution is at most 1.57 larger than the set of initial haplotypes. The ability of computing a good approximation seems affected by two combined factors: the number of independent characters of the genotype matrix and the number of initial haplotypes. Indeed in both tables we can observe that the smaller the number of independent characters compared to the number of initial haplotypes, the worse is the computed solution. Conversely good solutions are computed by the heuristic when the number of independent characters is close to the number of initial haplotypes.

Lemma 2.6 offers a possible explanation to such regular behavior of our heuristic. In fact, let $H$ be the set of initial haplotypes of an instance $X$ and suppose that they are defined on a set $\Sigma$ of independent characters such that $|H|=|\Sigma|+1$ (i.e. $H$ is also a solution that meets the lower bound of Lemma 2.6). Then, the set $T$ computed during step 7 of the heuristic algorithm contains exactly $|\Sigma|$ independent xor-genotypes. As a consequence, the set $C$ computed in the same step admits a Graph Realization and, thus, the heuristic solves optimally the instance $X$. Although this is not the general case, our intuition suggests that, when the number of independent characters is close to the number of initial haplotypes, the selection of the set $T$ is constrained and the Graph Realization of the maximal subset of $C$ computed by the heuristic is similar to the xor-graph associated with the initial haplotypes. Conversely, if the number of independent characters is significantly lower than the number of initial haplotypes, there are a lot of degrees of freedom in the choice of the set $T$, thus the output of the Graph Realization step can vary greatly from the xor-graph of the initial haplotypes.

The time required by the heuristic to compute a solution to the purerandom synthetic instances varies between circa 25 seconds on instances with 100 genotypes and 70 minutes on instances with 400 genotypes. All the instances generated using the neutral model, instead, have been solved in less than 1 minute. We also observe that instances where the heuristic fails to find a good solution have been solved considerably faster than the ones where the heuristic computes a good approximation. However, a more careful analysis suggests that such fluctuations are due to the different amount of I/O operations needed to communicate with the GREAL software that we use to solve the Graph Realization problem.

Finally we tried to compare our heuristic method with the ILP formulation proposed by Brown and Harrower [6]. In the paper, they formulate the PPXH problem as a polynomial-size integer linear program and they introduce cuts and modification of the objective function that should help finding the optimal solution. However, the GLPK solver [17], using the basic formulation as well as the augmented formulations, was not able to find a feasible solution even for the smallest instances of our experimentation (50 genotypes and 50 characters) within the maximum time of 24 hours. 


\subsubsection{Real Data}

To validate the feasibility of applying our heuristic on real data, we have produced some instances from the Phase I dataset of the HapMap project [19] (release 2005-06_16c.1). A set of xor-genotypes were produced from the data for each population in the dataset (discarding non biallelic sites and non autosomal chromosomes). Those instances vary from 44 genotypes and 184604 sites to 90 genotypes and 91812 sites. On average, an instance contains 67 genotypes and 46906 sites. On all those instances our heuristics has never required more than 2 seconds on the same PC used in the experimental part over synthetic instances, clearly establishing that the heuristic can be successfully used on real-world large instances.

\section{Conclusions and Future Work}

In the paper we investigate the problem of resolving xor-genotypes under the pure parsimony model. We give several results regarding the efficient solution of the problem by considering fixed-parameter algorithms or by restricting the instances of the problem. Most of the results are based on combinatorial properties of a graph representation relating a feasible solution to the instance: the xor-graph. The computational complexity of the unrestricted problem is still unknown. Since we show that $\operatorname{PPXH}(\infty, 2)$ and $\operatorname{PPXH}(2, \infty)$ have polynomial time algorithms, it would be interesting to determine the complexity of $\operatorname{PPXH}(\infty, 3)$ and $\operatorname{PPXH}(3, \infty)$, as these two cases could delimit polynomial time solvability and intractability of the general problem. We believe that the xor-graph could play a crucial role in solving these open problems.

\section{Acknowledgments}

PB, GDV and YP have been partially supported by FAR 2008 grant "Computational models for phylogenetic analysis of gene variations". PB has been partially supported by the MIUR PRIN 2007 Project "Mathematical aspects and emerging applications of automata and formal languages".

\section{References}

[1] T. Barzuza. GREAL - software for the graph realization problem.

[2] T. Barzuza, J. S. Beckmann, R. Shamir, and I. Pe'er. Computational problems in perfect phylogeny haplotyping: Xor-genotypes and tag SNPs. In Proc. 15th Symp. on Combinatorial Pattern Matching (CPM), volume 3109 of $L N C S$, pages 14-31. Springer, July 5-7, 2004. 
[3] T. Barzuza, J. S. Beckmann, R. Shamir, and I. Pe'er. Computational problems in perfect phylogeny haplotyping: Typing without calling the allele. IEEE Transactions on Computational Biology and Bioinformatics, 5(1):101-109, 2008.

[4] J. R. Bitner, G. Ehrlich, and E. M. Reingold. Efficient generation of the binary reflected Gray code and its applications. Communications of the ACM, 19(9):517-521, 1976.

[5] R. E. Bixby and D. K. Wagner. An almost linear-time algorithm for graph realization. Mathematics of Operations Research, 13:99-123, 1988.

[6] D. G. Brown and I. M. Harrower. Integer programming approaches to haplotype inference by pure parsimony. IEEE Transactions on Computational Biology and Bioinformatics, 3(2):141-154, 2006.

[7] R. Diestel. Graph Theory, volume 173 of Graduate Texts in Mathematics. Springer-Verlag, Heidelberg, third edition, 2005.

[8] R. Downey and M. Fellows. Parameterized Complexity. Springer Verlag, 1999.

[9] E. Fredkin. Trie memory. Communications of the ACM, 3(9):490-499, 1960.

[10] S. Fujishige. An efficient PQ-graph algorithm for solving the graph realization problem. Journal of Computer and System Science, 21:6368, 1980.

[11] F. Gavril and R. Tamari. An algorithm for constructing edge-trees from hypergraphs. Networks, 13(3):377-388, 1983.

[12] D. Gusfield. Haplotyping as perfect phylogeny: Conceptual framework and efficient solutions. In Proc. 6th Ann. Conf. on Research in Computational Molecular Biology (RECOMB), pages 166-175, 2002.

[13] D. Gusfield. Haplotype inference by pure parsimony. In Proc. 14th Symp. on Combinatorial Pattern Matching (CPM), pages 144-155, 2003.

[14] R. R. Hudson. Generating samples under a Wright-Fisher neutral model of genetic variation. Bioinformatics, 18(2):337-338, Feb. 2002.

[15] G. Lancia, M. C. Pinotti, and R. Rizzi. Haplotyping populations by pure parsimony: Complexity of exact and approximation algorithms. INFORMS Journal on Computing, 16(4):348-359, 2004. 
[16] G. Lancia and R. Rizzi. A polynomial case of the parsimony haplotyping problem. Operations Research Letters, 34(3):289-295, 2006.

[17] A. Makhorin. GLPK - the GNU Linear Programming Kit.

[18] C. Savage. A survey of combinatorial Gray codes. SIAM Review, 39(4):605-629, 1997.

[19] The International HapMap Consortium. A haplotype map of the human genome. Nature, 437(7063):1299-1320, 2005.

[20] W. T. Tutte. An algorithm for determining whether a given binary matroid is graphic. Proceedings of the American Mathematical Society, 11(6):905-917, 1960.

[21] L. van Iersel, J. Keijsper, S. Kelk, and L. Stougie. Shorelines of islands of tractability: Algorithms for parsimony and minimum perfect phylogeny haplotyping problems. IEEE Transactions on Computational Biology and Bioinformatics, 5(2):301-312, 2008. 


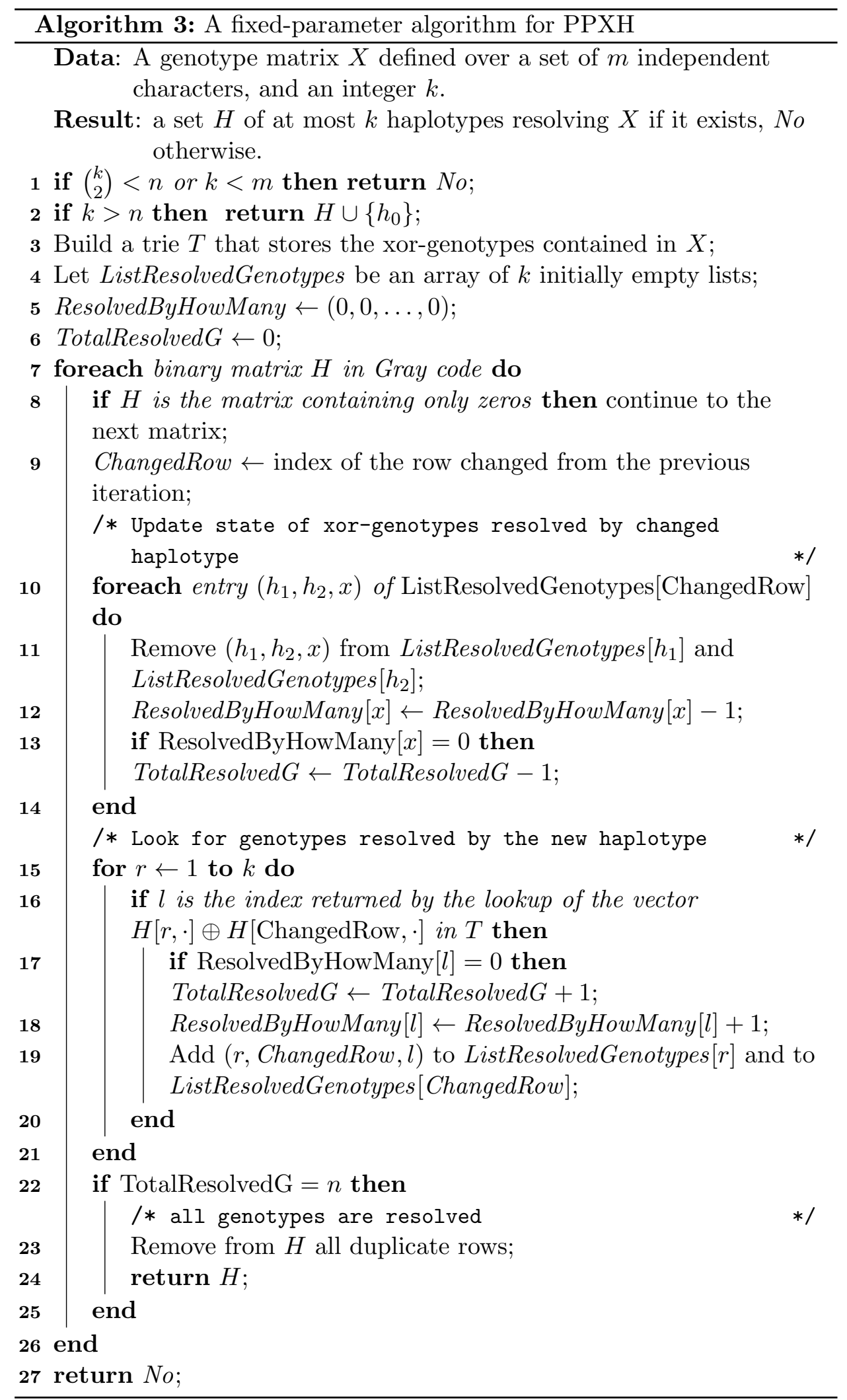



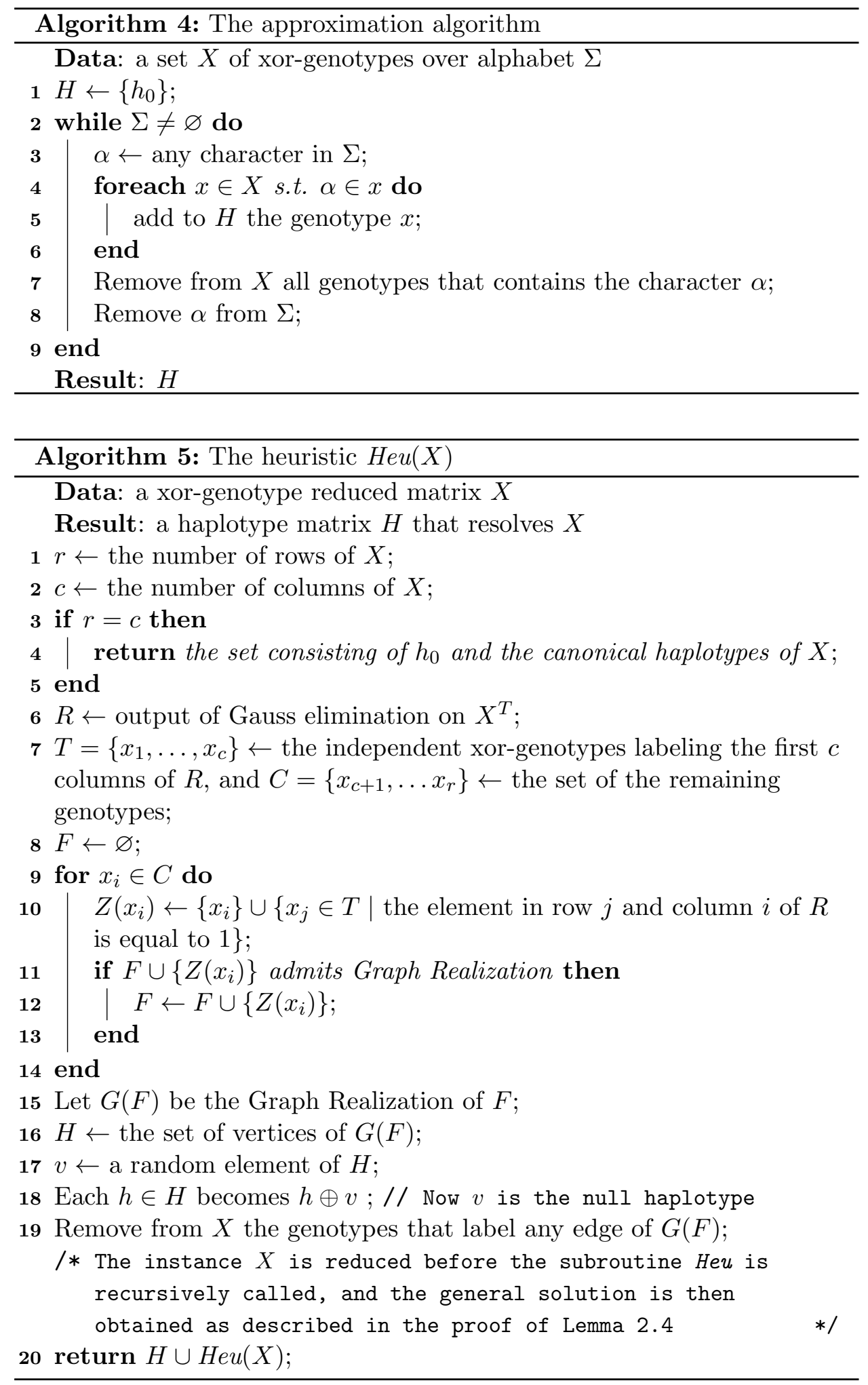
Table 2: Results on instances generated using the pure random strategy. For each choice of the first three columns, 10 random instances were generated. The column average independent characters reports the average number of independent character in the genotype matrix, while column average initial haplotypes reports the average number of distinct haplotypes selected to generate each instance. The last two columns report, respectively, the average size of the solution computed by our heuristic and the average ratio

\begin{tabular}{|c|c|c|c|c|c|c|}
\hline $\begin{array}{c}\text { number of } \\
\text { genotypes } \\
n\end{array}$ & $\begin{array}{c}\text { number of } \\
\text { generated } \\
\text { haplotypes } \\
h\end{array}$ & $\begin{array}{c}\text { number of } \\
\text { characters } \\
m\end{array}$ & $\begin{array}{c}\text { average } \\
\text { indepen- } \\
\text { dent } \\
\text { characters } \\
\end{array}$ & $\begin{array}{c}\text { average } \\
\text { initial } \\
\text { haplo- } \\
\text { types } \\
\end{array}$ & $\begin{array}{l}\text { average } \\
\text { result size }\end{array}$ & $\begin{array}{c}\text { average } \\
\text { ratio } \\
r\end{array}$ \\
\hline \multirow[t]{9}{*}{100} & 25 & 25 & 23.70 & 25.00 & 25.90 & 1.04 \\
\hline & & 33 & 24.00 & 25.00 & 25.00 & 1 \\
\hline & & 66 & 24.00 & 25.00 & 25.00 & 1 \\
\hline & 33 & 25 & 25.00 & 32.90 & 51.60 & 1.57 \\
\hline & & 33 & 31.50 & 32.80 & 33.20 & 1.01 \\
\hline & & 66 & 32.00 & 33.00 & 33.00 & 1 \\
\hline & 66 & 25 & 25.00 & 63.00 & 87.30 & 1.39 \\
\hline & & 33 & 33.00 & 63.30 & 87.20 & 1.38 \\
\hline & & 66 & 62.70 & 63.90 & 63.80 & 1 \\
\hline \multirow[t]{9}{*}{200} & 50 & 50 & 48.70 & 50.00 & 50.90 & 1.02 \\
\hline & & 66 & 49.00 & 50.00 & 50.00 & 1 \\
\hline & & 133 & 49.00 & 50.00 & 50.00 & 1 \\
\hline & 66 & 50 & 50.00 & 65.80 & 96.20 & 1.46 \\
\hline & & 66 & 64.50 & 65.90 & 66.20 & 1 \\
\hline & & 133 & 64.80 & 65.80 & 65.80 & 1 \\
\hline & 133 & 50 & 50.00 & 126.90 & 185.80 & 1.46 \\
\hline & & 66 & 66.00 & 126.20 & 186.10 & 1.47 \\
\hline & & 133 & 126.60 & 128.10 & 127.70 & 1 \\
\hline \multirow[t]{9}{*}{300} & 75 & 75 & 73.70 & 75.00 & 75.80 & 1.01 \\
\hline & & 100 & 74.00 & 75.00 & 75.00 & 1 \\
\hline & & 200 & 73.90 & 74.90 & 74.90 & 1 \\
\hline & 100 & 75 & 75.00 & 99.80 & 149.80 & 1.50 \\
\hline & & 100 & 98.60 & 99.90 & 100.00 & 1 \\
\hline & & 200 & 98.80 & 99.80 & 99.80 & 1 \\
\hline & 200 & 75 & 75.00 & 190.80 & 285.90 & 1.50 \\
\hline & & 100 & 100.00 & 191.10 & 284.60 & 1.49 \\
\hline & & 200 & 188.20 & 190.30 & 189.20 & 0.99 \\
\hline \multirow[t]{9}{*}{400} & 100 & 100 & 98.50 & 100.00 & 100.90 & 1.01 \\
\hline & & 133 & 98.90 & 99.90 & 99.90 & 1 \\
\hline & & 266 & 98.90 & 99.90 & 99.90 & 1 \\
\hline & 133 & 100 & 100.00 & 132.80 & 194.90 & 1.47 \\
\hline & & 133 & 131.40 & 132.80 & 133.00 & 1 \\
\hline & & 266 & 131.80 & 132.80 & 132.80 & 1 \\
\hline & 266 & 100 & 100.00 & 254.70 & 385.30 & 1.51 \\
\hline & & 133 & 133.00 & 253.60 & 384.40 & 1.52 \\
\hline & & 266 & 251.90 & 253.50 & 252.90 & 1 \\
\hline
\end{tabular}


Table 3: Results on instances generated using the neutral model. For each choice of the first three columns, 30 random instances were generated. The column average independent characters reports the average number of independent character in the genotype matrix, while column average initial haplotypes reports the average number of distinct haplotypes selected to generate each instance. The last two columns report, respectively, the average size of the solution computed by our heuristic and the average ratio

\begin{tabular}{|c|c|c|c|c|c|c|}
\hline $\begin{array}{l}\text { number of } \\
\text { genotypes } \\
n\end{array}$ & $\begin{array}{c}\text { number of } \\
\text { characters } \\
m\end{array}$ & $\begin{array}{l}\text { recombination } \\
\text { rate } \\
\rho\end{array}$ & $\begin{array}{l}\text { average } \\
\text { indepen- } \\
\text { dent } \\
\text { characters }\end{array}$ & $\begin{array}{l}\text { average } \\
\text { initial } \\
\text { haplo- } \\
\text { types } \\
\end{array}$ & $\begin{array}{l}\text { average } \\
\text { result size }\end{array}$ & $\begin{array}{c}\text { average } \\
\text { ratio } \\
r\end{array}$ \\
\hline \multirow[t]{12}{*}{50} & 50 & 0 & 18.5 & 19.5 & 19.5 & 1 \\
\hline & & 8 & 20.13 & 21.73 & 22.03 & 1.02 \\
\hline & & 16 & 22.27 & 24.77 & 25.77 & 1.04 \\
\hline & & 24 & 20.63 & 24.17 & 25.23 & 1.05 \\
\hline & 75 & 0 & 22.1 & 23.13 & 23.1 & 1 \\
\hline & & 8 & 24.63 & 26 & 26.27 & 1.01 \\
\hline & & 16 & 25.6 & 27.3 & 27.37 & 1.01 \\
\hline & & 24 & 25.3 & 27.63 & 28.1 & 1.02 \\
\hline & 100 & 0 & 25.07 & 26.13 & 26.07 & 1 \\
\hline & & 8 & 26.7 & 27.93 & 27.8 & 1 \\
\hline & & 16 & 28.27 & 29.87 & 29.73 & 1 \\
\hline & & 24 & 28.5 & 30.5 & 30.2 & 0.99 \\
\hline \multirow[t]{12}{*}{75} & 50 & 0 & 21.77 & 22.77 & 22.77 & 1 \\
\hline & & 8 & 23.1 & 25.37 & 26.63 & 1.05 \\
\hline & & 16 & 24.97 & 29.77 & 34.4 & 1.16 \\
\hline & & 24 & 25.1 & 31.4 & 38.33 & 1.23 \\
\hline & 75 & 0 & 26.17 & 27.2 & 27.17 & 1 \\
\hline & & 8 & 29.9 & 31.5 & 31.77 & 1.01 \\
\hline & & 16 & 30.93 & 34.63 & 37.1 & 1.07 \\
\hline & & 24 & 31.23 & 35.83 & 38.83 & 1.08 \\
\hline & 100 & 0 & 29.5 & 30.5 & 30.5 & 1 \\
\hline & & 8 & 32.87 & 34.23 & 34.13 & 1 \\
\hline & & 16 & 33.67 & 36.1 & 36.77 & 1.02 \\
\hline & & 24 & 36.2 & 39.73 & 41.17 & 1.04 \\
\hline \multirow[t]{12}{*}{100} & 50 & 0 & 24.33 & 25.33 & 25.33 & 1 \\
\hline & & 8 & 27 & 30.67 & 36.6 & 1.2 \\
\hline & & 16 & 27.53 & 32.8 & 41.8 & 1.28 \\
\hline & & 24 & 27.93 & 36.1 & 49 & 1.36 \\
\hline & 75 & 0 & 27.83 & 28.83 & 28.83 & 1 \\
\hline & & 8 & 32.2 & 34.4 & 36.07 & 1.05 \\
\hline & & 16 & 34.23 & 38.33 & 43 & 1.12 \\
\hline & & 24 & 35.23 & 42.07 & 50.43 & 1.2 \\
\hline & 100 & 0 & 34.5 & 35.5 & 35.5 & 1 \\
\hline & & 8 & 37.37 & 39.13 & 40 & 1.02 \\
\hline & & 16 & 39.87 & 43.27 & 45.63 & 1.06 \\
\hline & & 24 & 38.6 & 45.13 & 52.87 & 1.17 \\
\hline
\end{tabular}

